Preprint. Final version published in Origins of Life and Evolution of Biospheres April 2010, 40(2): 169177, http://www.springerlink.com/openurl.asp?genre=article\&id=doi:10.1007/s11084-010-9196-z

\title{
On what it is to fly can tell us something about what it is to live
}

\author{
Christophe Malaterre
}

\begin{abstract}
The plurality of definitions of life is often perceived as an unsatisfying situation stemming from still incomplete knowledge about 'what it is to live' as well as from the existence of a variety of methods for reaching a definition. For many, such plurality is to be remedied and the search for a unique and fully satisfactory definition of life pursued. In this contribution on the contrary, it is argued that the existence of such a variety of definitions of life undermines the very feasibility of ever reaching a unique unambiguous definition. It is argued that focusing on the definitions of specific types of 'living systems' - somehow in the same way that one can define specific types of 'flying systems' - could be more fruitful from a heuristic point of view than looking for 'the' right definition of life, and probably more accurate in terms of carving Nature at its joints.
\end{abstract}

Keywords Definition of life $\bullet$ Living systems $•$ Origin of life $\bullet$ Pluralism

\section{Introduction}

Definitions of life abound in the literature. Simultaneously, there is much debate among scientists and philosophers to find 'the' right definition of life. Yet, should we really worry about the existence of such a plurality of definitions and work towards a unique definition of life? In this contribution, I propose two arguments that account for such a diversity of definitions of life: a 'methodological argument' that has to do with the methods of definition, and a 'natural argument' stemming from research on physicochemical systems at the border-line between inanimate matter and life. I argue that, instead of searching for 'the' right definition of 'life', one ought to focus on defining types of 'living systems', for not only living systems might come with varying degrees of 'lifeness' but also with varying means for achieving this 'lifeness'.

C. Malaterre $(\square)$

Université Paris 1 - Panthéon Sorbonne, Institut d'Histoire et Philosophies des Sciences et Techniques, 13 rue du Four, 75006 Paris, France

e-mail: christophe.malaterre@gmail.com 


\section{On the many definitions of life}

The literature is replete with definitions of life. Already in the 1970s, Carl Sagan noticed that there was no commonly accepted definition of life and that there was a clearly discernible tendency for each biological discipline to define life in its own terms (Sagan 1970, 985); at that time, Sagan identified physiological, metabolic, biochemical, genetic and thermodynamic definitions of life. More recently, following the work of Palyi, Zucchi and Caglioti (2002, 15-56), Popa catalogued over ninety different definitions of life, from the 1850s to today (Popa 2004, 197-205). Some of these definitions are rather short, others much more intricate.

A closer look actually shows that they tend to fall into two broad categories. One finds 'list-based definitions' that define a living system in terms of a list of putative necessary and sufficient properties. Such properties typically include growth, reproduction, self-repair, energy-harnessing capability, matter-harnessing capability, variation capability, information capability and so forth (see for instance: Oparin 1961, Bernal 1967, Monod 1970, Crick 1981, Mayr 1982, de Duve 1991, Farmer and Belin 1992, Koshland 2002, Morange 2003). On the other hand, one also finds 'model-based definitions' that define living systems on the basis of a model that describes the very functioning of such living systems (see for instance: Maturana and Varela 1973, Ganti [1971] 2003, Ruiz-Mirazo, Pereto and Moreno 2004). In such cases, the properties of living systems are only secondary in the sense that they are nothing but a consequence of the functioning of the model. Whichever way one may adopt to classify definitions of life, the end-result is straightforward: definitions of life abound.

Such a situation is often perceived as being unsatisfactory: there is indeed much debate about the relative strengths and weaknesses of definitions of life when compared to one another, about the adequacy of such and such definition when it comes to capturing the 'essence' of life. There is also much debate about the existence of physical, chemical or biological counterexamples that seem to defy both the sufficiency and necessity of any of these definitions (e.g. Luisi 1998, Ruiz-Mirazo, Pereto and Moreno 2004). Many discussions focus on finding 'the' right definition of life. For instance, Cleland and Chyba argue that, in order to reach 'the' correct definition of life, one first needs to have a theory of life, somehow in the same way that one needs a molecular theory of water to correctly define water as $\mathrm{H}_{2} \mathrm{O}$ (Cleland and Chyba 2002). And, besides intellectual satisfaction, specific motivations for finding 'the' right definition of life are often put forward, including for instance use in astrobiology (to define whether one finds life or not elsewhere than on Earth), use in artificial life (to decide whether specific in silico systems do qualify as being alive or not), or use in synthetic biology (to decide whether specific 'wet' in vitro systems are alive or not).

In what follows, I argue that there are good reasons for such a plurality of definitions of 'life', and furthermore that the debate could benefit from tackling instead the question of defining 'types of living systems'.

\section{Definitional pluralism}

Two arguments can explain why there is such a plurality of definitions of life: first, a 'methodological argument' that borrows from logic and linguistics, and that concerns the art of defining; and second a 'natural argument' based on an analysis of the variety 
of functionalities that can be harnessed to sustain life-like processes ${ }^{1}$.

The 'methodological argument' stems from the simple observation that, there exist, in logic and linguistics, quite many methods for reaching definitions of terms, and that such methods may generate indeed quite distinct definitions of any given term. For instance, Clark and Welsh, in their logic manual (1962), list a number of such methods and types of definitions. One can first distinguish between 'lexical definitions' and 'stipulative definitions': whereas the first ones give or explain the meaning of a word by referring to the linguistic usage of this very word by certain people at certain places and times, the second ones deliberately assign a meaning to a word (Clark and Welsh 1962, 175-181). As a matter of fact, 'stipulative definition' is the principal sort of defining that is at stake when one claims that arguments can be settled by clarifying definitions, and, in this respect, the various definitions of life belong to this class of 'stipulative definitions'. But there are also many ways to elaborate a 'stipulative definition' of any given word. Clark and Welsh catalogue no less than eight methods of definition: the 'synonymous method', the 'method of analysis by genus and differentia', the 'relational or synthetic method', the 'denotative method', the 'range method', the 'ostensive method', the 'implicit method' and even the so-called 'regular method' (Clark and Welsh $1962,182-192)^{2}$. As a result, it is no surprise that multiple definitions of any given term might co-exist.

Indeed, the variety of methods of definition can certainly explain part of the observed plurality of definitions of life: for instance, the approach of many proponents of 'listbased definitions' of life definitely borrows from the 'method of analysis by genus and differentia' in so far as such definitions list specific properties that are supposed to set apart living systems from other types of physico-chemical systems; on the other hand, the approach of proponents of 'model-based definitions' of life is more akin to the 'relational or synthetic method' in so far as living systems are, in this case, defined by being related to particular models and their way of functioning.

The 'natural argument' stems from the diversity of physico-chemical systems that appear to populate the bordering zone between non-life and life. There is indeed quite a wide disagreement about whether certain systems are alive or not. One of the most frequently discussed cases is that of viruses. For some, viruses should not count as living systems in so far as they lack proper metabolic activity (see for instance Luisi 1998, Ruiz-Mirazo, Pereto and Moreno 2004). Yet, for others, they should: in

\footnotetext{
${ }^{1}$ It can also be argued, as a third argument, that 'life' is a folk concept that does not correspond to a natural kind, and that, as a result, it is no surprise that no right definition of life can be singled out (Machery, ms.). In this contribution, I will focus on a more scientific viewpoint.

${ }^{2}$ The 'synonymous method' consists in defining a word by giving another word which is its synonym ('chat' in French means 'cat' in English); the 'method of analysis by genus et differentia' consists in giving the name of a larger class (the genus) of which the thing to be defined is a subclass, and giving a property (differentia) which sets it off from other members of the larger class (a 'bachelor' is an 'unmarried adult man'); the 'relational or synthetic method' defines a word by relating the thing meant to some other thing we are familiar with (the color 'blue' can be correlated with a given set of light waves); the 'denotative method' proceeds by giving a list of examples of things the word applies to (a 'card game' is something like bridge, poker or twenty-one); the 'range method' defines a paradigm case and deviating borderline cases (a 'Labrador retriever' can be defined by presenting typical specimens and borderline specimens); the 'ostensive method' defines a word by presenting an object ('this is a thermometer'); the 'implicit method' defines a word by using it (the word 'hemisphere' can be defined by saying: 'the diameter of a circle cuts the circle into two equal hemispheres'); the 'regular method' defines a word by giving the rule for its use (the rule for ' $w e$ ' is that it is used by the speaker to refer to himself or herself and at least one other person). For more details, see for instance Clark and Welsh (1962, 182-192).
} 
particular, recent research is challenging the traditional view of a virus pictured as a rather static strand of nucleic acid encapsulated in a capsid, replacing it by that of a quite active and alive 'viral factory' (see for instance Forterre 2009, this volume). There is also substantial debate about whether self-replicating strands of RNA as hypothesized in the 'RNA world' scenario (Gilbert 1986) could count as being truly alive or not. For some, such an RNA strand would be capable of replication, possibly with variation thanks to replication errors, and therefore would be subject to natural evolution, hence alive (see for instance Luisi 1998). For others, on the contrary, self-replicating RNA strands would be far from qualifying as living systems: at best would they be selfreplicating and variation-capable systems; yet they would lack metabolic activity and would also not be enclosed in any membrane-like compartment (see for instance Shapiro 1986, Segré et al. 2001). Similarly, one can imagine the debate about the status of a physico-chemical system that would be capable of metabolic activity yet incapable of replication; an extreme example could be that of an organism that would comprise a planet in its entirety; yet another that of self-sustaining vesicles. One can also question whether a self-replicating system that would have a compartmentalization and a metabolic activity, but that would be devoid of any genetic code, might be called 'living' or not: most likely 'no' if one defines life on the basis of a genetic code, and 'yes' if one defines life on the basis of a metabolic activity. And the debate goes on about whether certain particular natural systems should be included or not within the restricted circle of 'living systems'.

What this debate illustrates, above all, is the wide range of properties and of ways of expressing these properties that might be called upon to define 'what it is to live'. Indeed, it appears today that the transition from 'inanimate matter' to 'life as we know it' is likely to be a very gradual process, involving multiple steps and spanning over millions, if not billions, of years (see for instance de Duve 1991). In this respect, there would therefore be no sharp point in time corresponding to the appearance of life: rather then resulting from a sudden emergence, 'life' is likely to be the outcome of a continuum of 'more-or-less alive' systems. Similarly, one can also argue that there still exist today physico-chemical systems that could be assessed as being more or less alive than other systems (as is the case, for instance, of viruses when compared to archae, bacteria or eukarya). In a sense therefore, there would be no clear-cut historical transition between non-living and living systems, nor any clear-cut delineation between current, be they natural or artificial, non-living and living systems. As a result, it can be argued that there are degrees of 'lifeness', that the property of 'being alive' or of 'pertaining to the class of living systems' is a matter of degree that cannot be captured within a framework resting on classical logic and the law of bivalence, but rather within a framework resting on fuzzy- or multi-valued logic (see Bruylants, Bartik and Reisse 2009, this volume): in such a case, physico-chemical systems may receive a score between 0 and 1 representing their degree of being alive. The upshot of the approach is to take into account the latest scientific knowledge about the origin of life, that is to say the very likelihood of a gradual transition from inanimate matter to life, and to formalize this continuum of 'lifeness'. Yet, the evaluation of any system along a 0 -to-1 scale seems to imply that being more-or-less alive is a question of degree along a onedimensional axis. Yet, is there indeed such a one-dimension along which to be 'moreor-less alive' or rather several dimensions, some of them potentially getting lost when projected onto a single axis?

As a matter of fact, a system can be more-or-less successful at replicating or 
reproducing itself, or more-or-less successful at metabolizing components or energy tokens from given sets of available nutrients and energy sources, or even endowed with a more-or-less sophisticated membrane. For instance, it appears very likely that, over time, membranes evolved very much in complexity: the abiotic synthesis of amphiphilic molecules, such as fatty acids, and their concentration and self-assembly might have resulted into vesicles, likely to be the first types of membranes encountered on the primitive Earth; yet such vesicles are very sensitive to concentration, temperature and pH (e.g. Monnard and Deamer 2002); the addition of other molecules such as sterols or amphiphilic polypeptides could have resulted, in a second step, in more robust vesicles, stable across varying chemical conditions, and larger as well (e.g. Luisi 2002); in a third step, the insertion of specialized transporters and active catalysts might have led to vesicles able to create and maintain chemical disequilibria; and the subsequent additions of other more complex organic compounds such as polysaccharides, energytransduction components or surface-layers components would have resulted in the appearance of the extremely sophisticated and multi-function membranes of current organisms (e.g. Ourisson and Nakatami 1994). One can therefore hypothesize the appearance of many different types of membranes of increasing complexity and performance. It might also be the case that only certain types of membranes are sophisticated enough to host life. In any case, the type of membrane that a particular physico-chemical system has might lead to qualify this system as more-or-less alive depending, in part, on the performance of this type of membrane: for instance, more-orless alive because of being more-or-less stable across varying chemical conditions, or because of being more-or-less capable of creating a chemical disequilibria etc. Similar performance scales could be put together for other major features of living systems: more or less efficient catalytic activity, more or less sophisticated metabolic pathways, more or less robust informational polymers etc. Such scales may then explain how a system might be more-or-less alive along several dimensions: for instance, a system might be capable of more-or-less successfully replicating, or more-or-less successfully metabolizing components. In addition, it might prove to be the case that in order to be, for instance, optimally replicating, one also needs to be successfully metabolizing, i.e. that in order to reach higher degrees of 'lifeness', then one needs to integrate and couple between themselves several of the above-mentioned dimensions. In sum therefore, it appears that the transition from inanimate matter to life is not a matter of a sudden jump but rather a matter of degrees, and that furthermore, such transition is not a matter of degrees along a single dimension but rather along several dimensions. As a result, defining 'life' becomes less relevant than defining specific 'types of living systems' that would characterize each of these dimensions and their inter-relatedness. Such an approach could also account for the current plurality of definitions of life. It is also commonplace in other fields of human enquiry. For instance, the multiple ways one can fly - more-or-less successfully! - have led to a plurality of definitions of the term 'aircraft'.

\section{Different types of 'what it is to fly'}

It seems one would know what flies and what doesn't, and that there is a clear-cut definition of 'what it is to fly': birds fly, as do aircrafts, but stones don't, neither do laptops. Yet, from paper airplanes to long-haul passenger aircrafts, there appears to be a 
wide range of performance and diversity of 'what it is to fly'. Dictionary-type definitions would say that to fly, in this semantic context, is "to move or be moved through the air by means of wings or a machine" ". Yet, such a generic definition has numerous counter-examples: hot-air balloons do not have wings nor a machine, neither do dandelion seeds, and yet they do fly. A similar problem arises when defining 'what it is to fly' as when defining 'what it is to live': stipulative definitions of such concepts fail to capture all necessary and sufficient conditions in which the terms should apply. Yet, a closer look at those 'flying systems' tells an interesting story.

In particular, the study of man-made flying systems - 'aircrafts' - reveals a great diversity of flying performances or 'degrees of successfully flying', to say the least ${ }^{4}$. For instance a propeller aircraft like the four-seat, single-engine, fixed-wing Cessna 172 Skyhawk, one of the most popular flight training aircraft in the world today, is likely to be qualified as a better flying system then the Blériot $n^{\circ} X I$ which crossed the Channel exactly one hundred years ago ${ }^{5}$ : both are single engine, fixed-wing aircrafts, yet the Cessna can carry more passengers, at a higher altitude and over longer distances than the Blériot could. One can therefore imagine a scale alongside of which could be measured the degrees of 'what it is to fly'. Such a scale would take into account a mix of flight performance parameters: distance, speed, cargo load or number of passengers, energy consumption, maintenance costs, durability, handling in adverse weather conditions etc.

A closer look at the story of aviation reveals that there is much more to "what it is to fly' than just a single one-dimensional flight performance scale. There are indeed several drastically different dimensions along which such performance could be measured, in short several ways of 'what it is to fly': one can glide; one can use a propeller to generate thrust and dynamic lift; or one can also take advantage of buoyancy. The existence of such a diversity of means of flying has led to a plurality of definitions of 'flying systems'. For instance, one can first distinguish between 'aerostats', such as hot-air balloons, and 'aerodynes', such as modern commercial airplanes: whereas 'aerostats' are lighter than air and can take advantage of buoyancy to achieve sustained movement through the air, 'aerodynes' are heavier than air and fly only by deriving lift from dynamic motion through the air.

Also, within 'aerodynes', one can distinguish those that are powered, such as propeller airplanes, from those are not, such as gliders. One can also distinguish those that have fixed wings from those that have flexible wings, or even those that have rotary wings: for instance, among 'unpowered aerodynes', one can find fixed-wing gliders, usually simply called 'gliders', but also flexible-wing gliders like so-called 'hang gliders' or 'paragliders', or even rotary-wing gliders like 'rotor-kites' or 'gyrogliders'.

A somehow similar, but more complex, classification holds for 'powered aerodynes'. In addition to using fixed-wings, flexible-wings or rotary-wings as means of providing lift, some 'powered aerodynes' might use other sources of lift, maybe more or less successfully. For instance, 'ornithopters' fly by flapping their wings somehow similarly

\footnotetext{
${ }^{3}$ See for instance the entry 'Fly' in Longman Dictionary of Contemporary English, Longman (1981).

${ }^{4}$ A similar argument could be made on the basis of naturally occurring flying systems, from birds to seeds, to even bacteria in cloud droplets (on the later, see for instance Sattler, Puxbaum, and Psenner 2001).

${ }^{5}$ Blériot crossed the Channel on July $25^{\text {th }} 1909$, reaching Dover from Calais in 37 minutes. See for instance Winchester (2007).
} 
to what birds $\mathrm{do}^{6}$; and aircrafts like the 'flying bedstead' relies on jet lift, with engine thrust directed downwards, as a means of providing lift ${ }^{7}$.

And there are also hybrid 'lighter-than-air/heavier-than-air' aircrafts. Among those are hybrid airships that combine characteristics of 'heavier-than-air' and 'lighter-thanair' technology, like helicopter/airship hybrids intended for heavy lift applications or dynamic lift airships intended for long-range cruising ${ }^{8}$.

This brief aeronautical survey illustrates not only that 'flying systems' can be moreor-less flying, i.e. that there are degrees of performance relative to flying, but also that flying can be achieved by quite different means and quite different arrangements of these means. The existence of some many different ways and degrees of flying has led to (1) a rather simple generic high-level definition of 'what it is to fly', accompanied by (2) dozens of more specific 'niche' definitions covering special ways and degrees of 'what it is to fly'. And, whereas the high-level definition captures the key features of flying without the ambition to fence off all possible counter-examples, the niche definitions specify much more precisely the types of 'flying systems', essentially on the basis of their functioning.

\section{Different types of 'what it is to live'}

In a similar way, the plurality of definitions of life could be accounted for by the existence of, not only performance degrees of living systems, but also the diversity of means and ways of functioning of such living systems. As a result, one should not worry so much about finding 'the' right definition of life: like the definition of 'to fly', the definition of 'to live' is likely to be prone to countless exceptions, no matter how well-crafted such definition might be: the reason is that there appears to be different ways thanks to which a chemical system might qualify as living. Instead, focusing on the definitions of more precise types of living systems - like one can define types of aircrafts - could prove to be more fruitful in terms of heuristics and more accurate in terms of carving Nature at its joints.

Indeed, different predicates can be defined to explicate 'what it is to live' when applied to particular types of physico-chemical systems. For instance, some of the major predicates may include:

- Replicating: property of a system that catalyzes the synthesis of systems that are identical to itself,

- Vario-replicating: property of a system that catalyzes the synthesis of systems that are nearly identical to itself (the 'nearly' could be further specified, for instance 95\% molecule-to-molecule identity),

- Metabolizing: property of a system that harnesses energy sources to produce chemical components while maintaining itself,

- Encoding: property of a chemical system that utilizes a molecular informational code,

\footnotetext{
${ }^{6}$ See for instance www.ornithopter.org

${ }^{7}$ This was for instance the case of the Rolls-Royce Thrust Measuring Rig of the 1950s, or the NASA Lunar Landing Research Vehicle of the 1960s.

${ }^{8}$ For instance, one can refer to the P-791 of Looked-Martin ("Lockheed Martin's Secretly Built Airship Makes First Flight", Aviation Week, 5 Feb. 2006) or the Aeroscraft project of Aeros (www.aerosml.com) among others.
} 
- Encapsulated: property of a chemical system that possesses a membrane which, in turn, provides a degree of isolation of the system from the environment, etc.

Accordingly, as a matter of illustration, the RNA-world would be populated by 'varioreplicating systems'; on the other hand, hydrothermal vents might have given birth to 'metabolizing systems'; and a current unicellular organism would qualify as an 'encapsulated metabolizing encoding and vario-replicating system'. Of course, the precise definitions of each predicate can be fine-tuned, as well as the list of these predicates. In addition, more fine-grained predicates could be devised to capture further distinctions between types of 'living systems', as scientific investigations reveal them. For instance, in the case of energy appropriation, one could define (on the basis of e.g. Popa 2004):

- Catalytic: property of a chemical system that catalyzes specific chemical reactions,

- Autocatalytic: property of a chemical system that catalyzes specific chemical reactions, some of which catalyze in turn the synthesis/restoration of the initial catalysts (reflexive activity),

- Energy-transducing: property of a chemical system that can transform one energy form into another energy form (and potentially store energy),

- Energy-regulating: property of a chemical system that can regulate the pace of energy flow.

Of course such definitions are still largely open and upcoming scientific research will tell which specific predicates are the most suited to describe the types of living systems (it is advances and failures in aeronautics that have revealed which ways of flying were possible and also more effective, and that have therefore shaped the predicates used to describe the great diversity of flying systems: aerostat/aerodyne, powered/unpowered, fixed-wing/flexible-wing/rotary-wing etc.). In any case, I argue that there are, at least, three major reasons for focusing on defining 'types of living systems', and no longer on defining the higher-level property of 'life'.

First, defining and explicating types of living systems should facilitate communication of scientific results, between scientists (in particular of different disciplines), as well as between the scientific community and the general audience (for instance in order to avoid hastily reached conclusions of the sort 'the secret of life has been discovered' or 'traces of life have been discovered on Mars'). This should also help laypersons realize how complex the transition from inanimate matter to life can be, and which key functions are required for a chemical system to gradually reach the performance of some of the most basic living systems.

Second, focusing on types of living systems and their key properties may have a heuristic value as it may help map out and investigate alternative ways of putting together life-contributing functions: after all, it is not by copying Nature and the flight of birds that man best managed to fly, but by devising a 'powered fixed-wing aircraft'; as a matter of fact, man-made ornithopters proved much more complex to build; furthermore, focusing on buoyancy instead of dynamic lift made possible the first manned free-flights in hot-air balloons a century before the first flights in "powered fixed-wing aircrafts" . Similarly copying Nature may not prove to be the best way for

\footnotetext{
${ }^{9}$ The first recorded free flight with human passengers onboard a hot-air balloon was on November 21, 1783 when de Rozier, along with Marquis Francois d'Arlandes, flew the balloon built by the brothers
} 
synthetic biology to make headway in creating novel in vitro living systems.

Third, such a pluralistic definitional strategy might also prove to be a more truthful way to carve Nature at its joints: the wide diversity of definitions of life as well as the numerous debates between scientists about the relative importance of specific sets of properties or models over others might indeed be interpreted as pointing, if anything, towards the existence of different ways and degrees of 'what it is to live'.

\section{Conclusion}

In this contribution, I proposed that two arguments can help explain the existence of a plurality of definitions of life: first a 'methodological argument' related to the diversity of possible methods of definition, and second a 'natural argument' stemming from the diversity of properties exhibited by border-line systems in between inanimate matter and life. Whereas, for some, the plurality of definitions of life is perceived as an unsatisfying situation that is to be remedied, I proposed that focusing, instead, on defining types of living systems might prove more fruitful and accurate as a way of accounting not only for different degrees of 'lifeness' or 'living performance', but also of accounting for the different means that physico-chemical systems might manage to harness in order to live. Like flying, living might indeed come not only in different intensities but also in different flavors.

Acknowledgments I am very grateful to the participants to the Defining Life conference organized in Paris in February 2008, for very stimulating discussions. Support from the CNRS interdisciplinary program "Origines des planètes et de la vie" and from the Fondation Louis D. of the Institut de France is gratefully acknowledged.

\section{References}

Bernal JD (1967) The Origin of Life. Weidenfeld and Nicolson, London

Bruylants GK, Bartik, Reisse J (2009), Is It Useful to Have a Clear-Cut Definition of Life? On the Use of Fuzzy Logic in Prebiotic Chemistry. Orig Life Evol Biosph (this volume)

Clark R, Welsh P (1962) Introduction to Logic. D. Van Nostrand, Princeton NJ

Cleland C, Chyba CF (2002) Defining Life. Orig Life Evol Biosph 32: 387-393

Crick F (1981) Life Itself: Its Origin and Nature. Simon \& Schuster, New York

De Duve C (1991) Blueprint for a cell : The Nature and Origin of Life. Patterson, Burlington NC

Farmer JD. and Belin A (1992) Artificial Life: The coming evolution, In: CG Langton, C Taylor, JD

Farmer, S Rasmussen (eds.), Artificial Life II, Santa Fe Institute Studies in the Sciences of Complexity Proceedings, Vol. X, Redwood City, CA: Addison-Wesley, pp. 815-838

Forterre P (2009) Defining Life: the Virus Viewpoint. Orig Life Evol Biosph (this volume)

Ganti T ([1971] 2003) The Principles of Life. Oxford University Press, Oxford

Gilbert W (1986) The RNA world. Nature 319: 618

Koshland DE Jr (2002) The seven pillars of life. Science 295: 2215-2216

Luisi PL (1998) About various definitions of Life. Orig Life Evol Biosph 28: 613-622

Luisi PL. (2002) Emergence in chemistry: chemistry as the embodiment of emergence. Foundations of Chemistry 4: 183-200

Montgolfier in Annonay, France. On the other hand, the Wright brothers made the first sustained, controlled, powered heavier-than-air manned flight at Kill Devil Hills, North Carolina, USA on December 17, 1903. See for instance Winchester (2007). 
Machery E (ms), Why I Stopped Worrying about the Definition of Life... And Why You Should as Well, (work in progress, personal communication)

Maturana H, Varela F (1973) Autopoiesis: The Organization of the Living. Dordrecht

Mayr E (1982) The Growth of Biological Thought. Diversity, Evolution, and Inheritance. The Belknap Press, Cambridge MA

Monnard P.-A, Deamer DW (2002) Membrane Self-Assembly Processes: Steps Toward the First Cellular Life. The Anatomical Record 268: 196-207

Monod J ([1970] 1971) Chance and Necessity. Vintage, New York

Morange M (2003) La vie expliquée ; 50 ans après la double hélice. Odile Jacob, Paris

Oparin AI (1961) Life: Its Nature, Origin and Development. Academic Press, New York

Ourisson G, Nakatani Y (1994) The terpenoid theory of the origin of cellular life: the evolution of terpenoids to cholesterol. Chemistry and Biology 1: 11-23

Palyi G., Zucchi, C, Caglioti L (eds.) (2002), Fundamentals of Life. Elsevier, Paris

Popa R (2004) Between Chance and Necessity: Searching for the Definition and Origin of Life, SpringerVerlag.

Ruiz-Mirazo K, Pereto J, Moreno A (2004) A universal definition of life: autonomy and open-ended evolution. Orig Life Evol Biosph 34(3): 323-346

Sagan C ([1970] 1986) Life. Encyclopedia Britannica, 15 ${ }^{\text {th }}$ ed., Vol. 22, Encyclopedia Britannica Inc., Chicago, pp 985-1002

Segré D, Ben-Eli D, Deamer DW, Lancet D (2001) The lipid world. Orig Life Evol Biosph 31: 119-145

Shapiro R (1986) Origins : A Skeptic's Guide to the Creation of Life on Earth.. Summit Books, New York Winchester J (2007) The Timeline of Aviation. Thunder Bay Press 